\title{
Embodiment and abstraction: Actions create relational representations
}

\author{
Jeremiah J. Trudeau and James A. Dixon \\ University of Connecticut, Storrs, Connecticut
}

\begin{abstract}
Theories of embodied, modal representation propose that concepts are grounded in the sensorimotor system. According to these theories, action (or the potential for action) creates meaning. An apparent challenge for these theories is the fact that higher-order relations (i.e., relations among relations), such as monotonic increase or alternation, have no straightforward sensorimotor analog. A single action can increase or alternate only relative to another action. Therefore, if embodied theories are to handle concepts more generally, patterns of action must provide meaning to the system. Previous research suggested that as participants simulated the motion of gear problems they discovered a higher-order relation, alternation, based on the episodic traces of their own alternating actions. The present study showed that the number of alternating actions in episodic memory prior to discovery of the alternation relation predicted its generalization to new problem types. Actions can function as the representational substrate of higher-order relations.
\end{abstract}

Recent theories of representation propose that conceptual knowledge is grounded in the various sensorimotor systems (Barsalou, 2005; Gallese \& Lakoff, 2005). Rather than locating concepts in a centralized, amodal semantic system, these theories suggest that representations are directly tied to the sensory modalities. Barsalou (2005) hypothesized that each sensory modality has an adjacent local memory layer which captures the activation patterns of particular objects, relations, or actions. The meaning of the object (or relation or action) - the system's knowledge about its specific properties - is accessed by reactivating the original patterns across the various relevant sensory modalities.

Previous work focused on predictions of the modal hypothesis for language comprehension. For example, Solomon and Barsalou (2001) showed that the degree to which verifying a property (e.g., "mane") for one concept (e.g., "horse") facilitated verifying that property for a second concept (e.g., "mane" for "pony") depended on whether the form of the property was physically similar for both concepts. Behavioral and cognitive neuroscience research supports the conclusion that comprehension involves the activation of relevant sensorimotor systems (Buccino et al., 2005).

Kaschak and Glenberg (2000) proposed that reactivation of sensorimotor areas results in perceptual representations; these representations have affordances in the same way that objects in the external environment do. The meaning of a representation activated during reading is derived from the potential actions specified by the affordances. For example, they showed that participants' comprehension of novel, denomial verbs (e.g., "crutching" a ball) involved detecting the affordances of objects described in the passages.

\section{Action Creates Meaning}

Affordances, in this account, specify the potential for action, given one's particular body situated in an environment. Action creates meaning, both through affordances (i.e., potential action) and physical movement - action in real time and space. Glenberg and Kaschak (2002) showed that reading a sentence which implied an action interfered with participants' ability to respond with an opposing action. For example, the sentence, "Close the drawer," implies movement away from the body. Participants were slower to verify such sentences as sensible, if the "Yes" button was positioned such that it required movement toward the body.

Zwaan and Taylor (2006) showed a similar actioncompatibility effect. Participants read sentences that implied rotation (e.g., "He turned down the volume") and then judged the sensibility of the sentences by turning a knob clockwise or counterclockwise. Responses were faster when the direction of the implied rotation matched the direction of the rotation response. They also demonstrated that visually presented rotation information created motor resonance, as evidenced by effects on manual rotation, and comprehension of sentences that imply rotation. Sensorimotor resonance provides meaning for language and perhaps visually presented motion.

Action also may ground cognition in reasoning and problem solving. For example, reasoning about depicted mechanical systems is facilitated by simulating appropriate actions (Hegarty, 2004). Interestingly, even when actions are not overtly in evidence, the time course of reasoning about these systems is consistent with an action-based representation. Grant and Spivey (2003) showed that eyemovement patterns on the classic Duncker radiation prob-

J. J. Trudeau, jaytrudeau@gmail.com 
lem were related to reaching the insightful, converging rays solution. This suggests that the action of performing eye movements may be fundamental to forming a new spatial representation. In sum, embodied accounts of representation posit that meaning is grounded in action.

\section{Relations in Amodal Systems}

Amodal, symbolic theories explain representation in a very different way. According to amodal theories, relations in the environment are encoded into a system of mental symbols. Computation over the relevant symbols results in a decision that is passed to motor programming areas which specify the appropriate actions (cf. Newell, 1990). Actions have environmental consequences (e.g., produce errors) that may be detected by the perceptual system, but the actions themselves are downstream, computational products of representations, rather than foundational for representation. Because actions are the products of representations here, it would be strange for them to contain information beyond that found already in the representation, or to create new representations.

Therefore, a core difference between modal and amodal approaches to representation is the posited role of action. In modal theories, actions create meaning; in amodal theories actions are the consequences of manipulating meaningful symbols.

\section{Embodiment of Higher Order Relations}

Embodied, modal accounts have considerable appeal for explaining the representation of first-order relations. For example, movement away from one's body and rotating an object have straightforward sensorimotor implications. However, explaining the representation of higher order relations appears to pose a serious challenge for embodied representation.

How might higher-order relations such as recursion, monontonic increase, or alternation enter a cognitive system that derives meaning from action? The difficulty is that none of these higher-order relations can be represented by a single action, because they require coordinating two or more lower-order relations. Alternation, for instance, specifies that adjacent objects in a series take different values of the relevant property and that this lower order relation is repeated across the series. A particular motion (e.g., pointing upward) is part of alternation by virtue of its relation to a prior motion (e.g., pointing downward). Therefore, alternation cannot be represented by any single action. Rather, representing this relation would require integrating multiple actions over time. The same logic applies for all higher-order relations because, by definition, they specify relations among relations. Therefore, if modality-specific systems are to handle higher order relations, they must be capable of summarizing action patterns into representations. The present article provides initial evidence regarding the grounding of higher order relationships in action.

\section{Alternation From Action in the Gear-System Task}

Dixon and Bangert (2002) investigated changes in participants' representation of a gear-system task. For each gear problem, participants predicted the turning direction of the target gear, given the turning direction of the driving gear which provided the force to the system (see Figure 1). Most participants first represented the gear system in terms of local, physical forces. They initially traced the force through the system by motioning with one hand. That is, they simulated the turning of driving gear, the pushing of the driving gear's teeth on the teeth of the next gear, the turning of that gear, and so on, ultimately establishing the turning direction of the target gear. When done correctly, tracing the force through the system results in actions that alternate direction; the direction of movement (i.e., clockwise, counterclockwise) changes from gear to gear.

After repeatedly tracing the force across the gears, many participants spontaneously stopped simulating the forces within the system and began sequentially classifying the gears as "counterclockwise" or "clockwise." That is, they began categorizing the gears as members of an alternating series, without reference to the physical forces. These participants appear to have spontaneously formed a new representation of the gear system - the gears alternate direction. Importantly, the gear displays were static; only the target gear actually moved. Therefore, the only source of information about the alternating nature of the system was the participant's own actions.

Dixon and Bangert (2002) proposed that the discovery of alternation (i.e., gears alternate) emerged from the activation of instances of force-tracing in episodic memory. They showed that discovering alternation depended on the interaction between (1) the participant's history of correctly using force-tracing across all previous trials, and (2) the proportion of force-tracing used on recent trials. The first predictor captures the degree to which a participant's episodic traces contain alternation information (incorrectly tracing the force does not produce alternating motions). The second predictor indexes the degree to which force-tracing instances were activated; concentrated use of force-tracing should strongly activate stored episodic traces. The probability of discovering alternation increased dramatically when both these factors increased.

Although college students probably understand alternation within other domains prior to engaging in the gearsystem task, evidence suggests that they do not readily transfer alternation to this domain. For example, Dixon and Dohn (2003) directly instructed participants to use the alternation strategy on a structurally analogous task in which balance beams were connected end-to-end in a series. Participants predicted whether the last balance beam in the series would go up or down, given the movement of first beam. After being instructed on alternation and solving 10 problems using alternation, participants were given gear-system problems. Despite their immediately prior use of the alternation strategy, participants showed no evidence of transfer - their median discovery trial did not differ from that of uninstructed participants. Thus, highlighting their knowledge of alternation and demonstrating its application within a similar task context did not produce transfer. This finding squares with a large body of work showing that transfer to a domain with unique surface features is very difficult to achieve (e.g., Catrambone, 2002). ${ }^{1}$ 

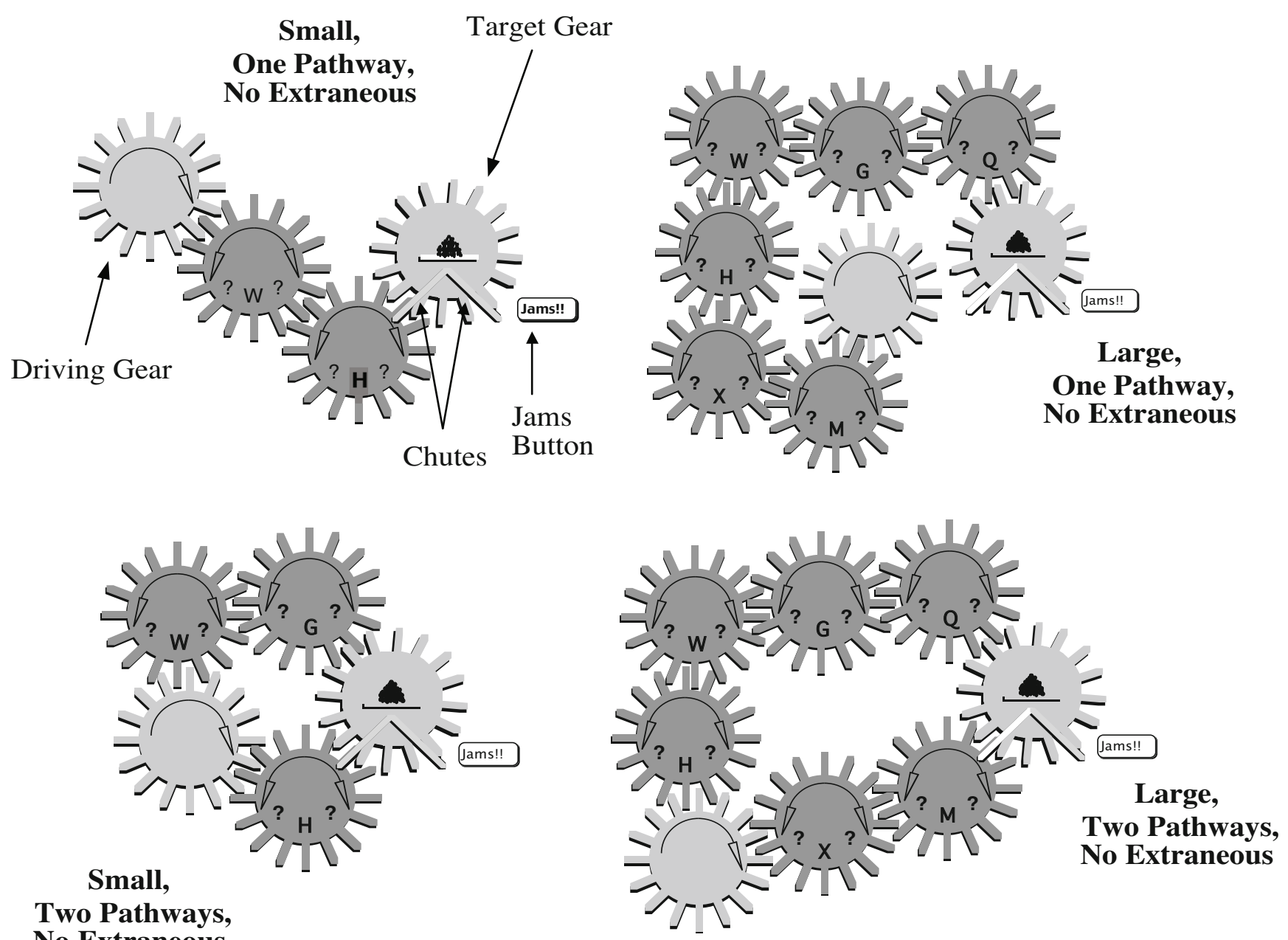

Two Pathways, No Extraneous

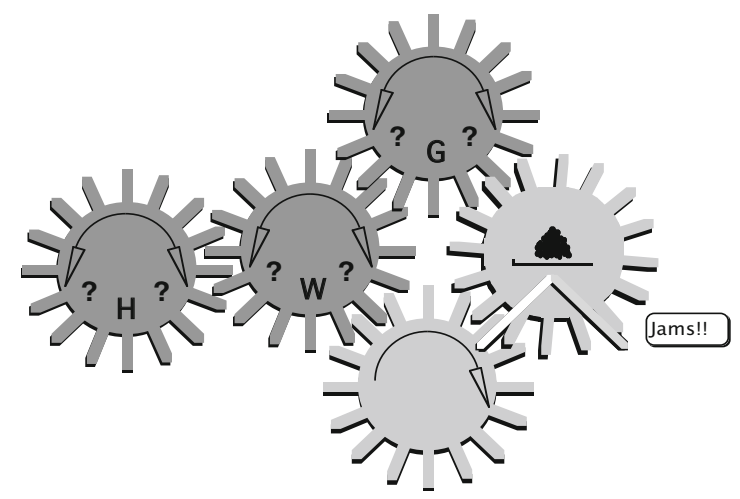

\section{Small, \\ Two Pathways, Extraneous}

Figure 1. Examples of different types of gear-system problems. The driving gear, with the single-headed arrow, provided the force to the system. Participants were asked to predict the turning direction of the target gear - the gear with the small pile of "fuel" on its face. They responded by clicking on one of the chutes on the target gear to indicate which way the fuel would fall or the jams button to indicate that the system would not turn. The types of systems are described by their values on each of three dichotomous dimensions: size, number of pathways, and presence of an extraneous gear.

\section{Episodic Traces of Actions and Generalization}

If the episodic traces of alternating actions are summarized into a representation of alternation, then the quality of the resulting representation should be a function of the number of relevant actions in memory. As the number of action traces increases, idiosyncratic contextual features of any particular problem experience (e.g., the spatial configuration of the gears) will become balanced against the features of other problem experiences (Goldinger, 1998; Hintzman, 1986). Features common to all the instances 
should emerge in sharper relief when the representation results from the activation of a larger corpus of traces. Therefore, participants who have made more correct alternating actions prior to consolidating those actions into a representation of alternation, will have a more abstract, generalizable representation of the relation.

The present study thus extends previous work, which focused on discovery of alternation, by testing predictions about generalization postdiscovery. We modeled the probability of generalizing alternation as a function of the number of alternating motions (i.e., the number of individual gears over which force-tracing was correctly performed) prior to discovering alternation. Participants who had discovered alternation were "at risk" for generalization each time they encountered a problem that differed (on at least one dimension) from the problem on which they had discovered alternation. (See Figure 1). If the representation of alternation is compiled from the episodic traces of action patterns, the number of alternating actions contributing to that representation should predict generalization.

\section{METHOD}

\section{Participants}

Seventy-six college students, who had discovered alternation during the gear-system task, and therefore were at risk for generalizing to different problem types, contributed data for this study. Participants were from three sources, Dixon and Bangert (2002), Dixon and Dohn (2003), and a new sample ${ }^{2}(n s=26,44$, and 6, respectively). Participants completed the experiment as one option to earn extra credit for a course.

\section{Procedure}

The task was presented as a computerized train race. By obtaining fuel at stations along the race course, participants could make their train go faster than the computer's train. Each fueling station was a gear system. The fuel, a pile of coal, was located on a shelf on the target gear. Participants predicted whether the target gear would turn clockwise or counterclockwise or jam (fail to turn because of opposing forces). Correctly predicting the outcome increased the speed of the participant's train, incorrect predictions decreased the speed.

A clockwise-turning, driving gear provided the force to each system. The driving gear was connected to the target gear by a series of intermediate gears. Two chutes were shown below the shelf on the target gear. Participants clicked on a chute to indicate which way they thought the fuel would fall when the gear turned. If the participant thought the target gear would not turn, he or she was to click on the jams button. Each of these actions (i.e., clicking on a chute or the jams button) positioned the participant's train appropriately (i.e. under the selected chute or ready to leave the nonturning station). Participants' indicated that they were satisfied with their solution by clicking on the train.

Clicking on the train caused a virtual screen to cover all the gears except the target gear. The target gear then turned appropriately given the physics of the system. Participants next saw a series of feedback events (e.g., speeding out of the station given a correct judgment). Participants were asked to think aloud during their solution attempts and their strategies were coded.

\section{Materials}

Gear systems varied along three dimensions: size (small, 4-5 gears; large, 7-8 gears), number of pathways between the driving and target gears (one or two pathways), and whether an extraneous gear was present (see Figure 1). Participants in the Dixon and Bangert (2002) sample solved 44 trials, all others solved 32 trials. The task was presented on a Macintosh PowerPC running SuperCard 3.0.

\section{RESULTS}

\section{Performance Prior to Discovering Alternation}

Strategy use and accuracy. Prior to discovering alternation, participants used the force-tracing strategy on $91.6 \%$ of the trials. The other strategies employed were: recalling an answer from a previous problem (2\%), using the parity of the number of gears to determine the turning direction of the target gear (3\%), unclassifiable or idiosyncratic approaches (3\%). ${ }^{3}$ Accuracy on these early trials was high; participants correctly predicted the target gear's motion on $92 \%$ of trials. Accuracy was unrelated to strategy type $\left[\chi^{2}(4)=4.39\right.$, n.s. $](\alpha=.05$ for all tests $)$.

Force-tracing actions. Prior to discovering alternation, participants generated alternating actions as a consequence of correctly tracing the force across the system. The mean number of correct alternating actions - the number of gears over which force-tracing was correctly performed prior to discovering the alternation relationwas $37.5(S D=31.67)$.

\section{Predicting Generalization of Alternation}

Most participants discovered alternation fairly quickly. Approximately $50 \%$ of participants discovered alternation by trial $8 ; 75 \%$ discovered by trial 13 . After discovering alternation, participants were at risk for generalization whenever the current problem was different from the problem on which they had discovered alternation.

Event history analysis. We modeled the probability of generalizing alternation postdiscovery using event history analysis (Singer \& Willett, 2003). In brief, event history analysis models the conditional probability of discrete events occurring over time. Two concepts are central to understanding the analyses presented here. First, the risk set is the group of all participants who have not yet experienced the event of interest (e.g., generalization of alternation). The risk set changes across trials. Participants who have experienced the event on a previous trial are dropped from the risk set; they no longer contribute to the analysis. Second, the hazard rate is the probability of the event occurring on a given trial. For each trial, the hazard rate is estimated by the number of participants in the risk set who experienced the event on that trial divided by the total number of participants currently in the risk set. Models were fit with logistic regression.

Because these participants discovered alternation during the experiment, we know the attributes of their discovery problem on the dimensions described above. After participants used alternation for the first time, they were at risk for generalization each time they encountered a problem that differed from their discovery problem on at least one dimension. For example, a participant who discovered alternation on a small, single pathway system with an extraneous gear would be at risk for generalization each time he or she encountered a system with that was large, had two pathways, or did not have an extraneous gear. 
Episodic action traces. We hypothesized that the number of episodic traces of alternating actions would predict generalization of the alternation relation. Consistent with current models of episodic memory (e.g., Goldinger \& Azuma, 2004; Logan, 2002), we assumed that episodic traces of all previous alternating actions were activated by making alternating actions on the current trial. We further assumed that the alternation information resulting from this activation, the episodic "echo" from probing memory (Goldinger, 1998; Hintzman, 1986), was then stored as a trace itself. Therefore, the measure of action traces was the sum of the number of gears over which force tracing had been correctly performed on all trials prior to discovery and the sum of all previous echos. This measure of the number of action traces that had contributed to the representation of alternation, $M=186.88(S E=18.8)$, was the crucial predictor of generalization. ${ }^{4}$

Modeling the probability of generalization. The curve with triangular markers in Figure 2 shows the probability of generalizing alternation as a function of the number of risk trials. The probability of generalization was high on the initial risk trial (i.e., the first trial that differed from the participant's discovery trial) and decreased nonlinearly across subsequent risk trials. Given this curvilinear form, we modeled the probability of generalization as a quadratic function of risk trial by including both the linear and squared terms (i.e., risk trial and risk trial ${ }^{2}$ ), $\left[B \mathrm{~s}=-.349, .067 ; S E \mathrm{~s}=.067, .017\right.$; Wald $\chi^{2}(1) \mathrm{s}=$ $26.89,14.82$, respectively].

We added the measure of action traces to the model as a linear term and in interaction with risk trial; both terms significantly improved the fit of the model, change in $-2 \mathrm{LL}$ $\left[\chi^{2}(1)=5.20,4.95 ; B \mathrm{~s}=.028, .008 ; S E \mathrm{~s}=.013, .004\right.$, respectively]. ${ }^{5}$ The linear coefficient indicates that partici-

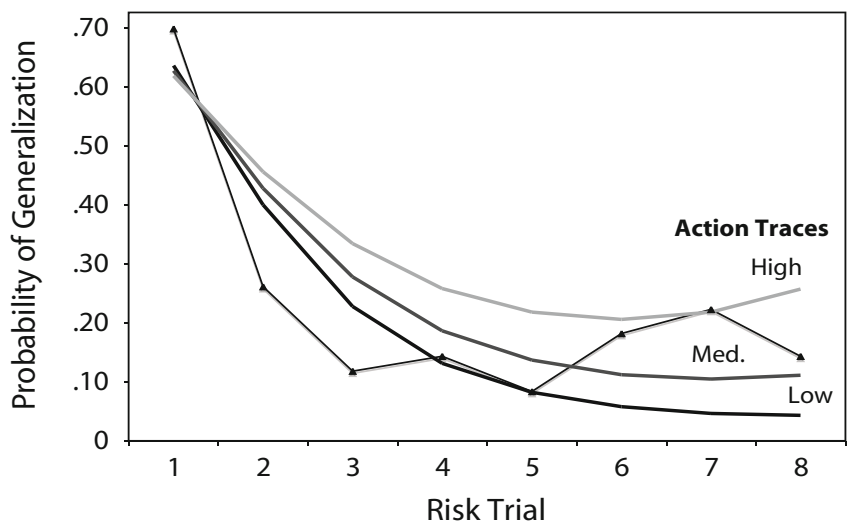

Figure 2. The line with the triangular markers shows the observed probability of generalization plotted as a function of risk trial. Participants were at risk for generalization when the current problem differed from their discovery problem on at least one dimension. Thus, the first risk trial was the first trial on which they could generalize, the second risk trial was the second time they were at risk (conditional on not having generalized on the first risk trial), etc.,. The smooth curves show the logistic regression results from the event history analysis. The effect of the number of action traces is illustrated by the three different curves for high $(+1 S D)$, medium (Mean), and low $(-1 S D)$ values of the action traces measure. pants with a larger corpus of action traces had a higher probability of generalizing alternation. The interaction between action traces and risk trial indicates that the probability of generalization over risk trials decreased less for participants who were higher on the action-traces measure. These regression results are illustrated in Figure 2. The probability of generalization is shown as a function of risk trials with separate curves for low, medium, and high action traces. The regression results show that participants who performed more alternating motions had a higher, overall probability of generalization, and that the probability of generalization decreased less rapidly for these participants. ${ }^{6}$

\section{DISCUSSION}

All participants in the present sample discovered alternation during the course of the study. However, prior to discovery, the number of alternating actions they produced while simulating the motions of the gears varied considerably. Previous work suggested that the episodic traces of these alternating actions were consolidated into a representation of alternation (Dixon \& Bangert, 2002; Dixon \& Dohn, 2003). The present results showed that participants whose representation of alternation was based on a larger episodic corpus of alternating actions generalized alternation to different types of problems more easily. Participants who had made more alternating actions prior to discovering alternation had a higher probability of generalizing alternation; further their probability of generalization decreased less sharply over time.

Therefore, the present study provides initial evidence that actions can function as the representational substrate of higher order relations. An individual's history of producing actions predicts both the formation and generalization of alternation in real-time. These results complement previous work (cited earlier) on the embodied, modal representation of concepts in which actions and affordances were shown to be involved in the comprehension of firstorder relations. Our findings demonstrate that actions can also ground higher order relations - relations that cannot be instantiated in a single motion. Thus, embodied, modal theories of representation may be capable of addressing a very broad class of concepts.

How might existing amodal theories accommodate these results? Most models of representational change propose that new representations are formed in response to error (see Kalish, Lewandowsky, \& Davies, 2005). As errors accumulate, the system draws on information in memory or the environment to replace or refine the current representation. Actions can trigger the search for a new representation, but they do not provide the informational basis for that representation. Thus, these accounts would require substantial modification to explain the present results - successful, rather than errorful, actions predict the quality of the new representation, as indexed by the generalizability of the representation.

A few existing theories propose that new representations arise from successful, as opposed to errorful, performance. In these theories, new representations or strategies are formed by recombining the components of existing 
strategies. These new strategies then compete probabilistically with previous strategies. For example, Siegler and Araya (2005) proposed that an associative system tracks the speed and accuracy of each strategy across various problem types. A metacognitive system examines the components of each strategy and invents new strategies by recombining those components according to some simple heuristics. New strategies are given a strong initial weight to allow them a chance to be selected over their successful, heavily weighted predecessors. This account has difficulty explaining the present results because it predicts generalization of alternation should be slow and negatively related to the number of force-tracing strategy uses.

An embodied approach to representation, particularly one in which actions play a central role, has implications for core issues in cognitive science. For example, on the standard account, the acquisition of new knowledge seemingly requires prior knowledge of which feature dimensions to encode. However, if representations are formed from actions, rather than amodally encoded features, this problem largely disappears because actions create the information. When the perception-action system operates on the affordances in the environment, the resulting actions become part of episodic memory. Given sufficient activation of these episodic traces, regularities among the actions emerge as new relational knowledge. For example, in the gear task, participants first respond to the local turning and pushing affordances, resulting in motions that simulate the force across the system. Information created by these alternating actions accumulates in episodic memory. Activation of the episodic traces consolidates this latent information into a representation of alternation. In this way, actions based on local affordances, the turning and pushing of individual gears, yield information about the system at higher temporal scale. Encoding features of the environment is not an issue, because the information is created from the interaction between the perception-action system and the environment, that is, through action. Thus, embodied approaches may provide new insight into knowledge acquisition and other fundamental problems in cognition.

\section{AUTHOR NOTE}

Correspondence concerning this article should be addressed to J. J. Trudeau, Department of Psychology, 406 Babbidge Road, Unit 1020, University of Connecticut, Storrs, CT 06269-1020 (email: jaytrudeau@ gmail.com).

\section{REFERENCES}

BARsalou, L. W. (2005). Abstraction as dynamic interpretation in perceptual symbol systems. In L. Gershkoff-Stowe \& D. Rakison (Eds.), Building object categories (pp. 389-431). Mahwah, NJ: Erlbaum.

Buccino, G., Riggio, L., Melli, G., Binkosfski, G., Gallese, V., \& Rizzolatti, G. (2005). Listening to action-related sentences modulates the activity of the motor system. Cognitive Brain Research, 24, 355-363.

Catrambone, R. (2002). The effects of surface and structural feature matches on the access of story analogs. Journal of Experimental Psychology: Learning, Memory, \& Cognition, 28, 318-334.

Dixon, J. A., \& BAngerT, A. (2002). The prehistory of discovery: Precursors of representational change in solving gear-system problems. Developmental Psychology, 38, 918-933.

Dixon, J. A., \& DoHn, M. C. (2003). Redescription disembeds relations:
Evidence from relational transfer and use in problem solving. Memory \& Cognition, 31, 1082-1093.

Gallese, V., \& LAKoff, G. (2005). The brain's concepts: The role of the sensory-motor system in conceptual knowledge. Cognitive Neuropsychology, 22, 455-479.

Glenberg, A. M., \& KaschaK, M. P. (2002). Grounding language in action. Psychonomic Bulletin \& Review, 9, 558-565.

Goldinger, S. D. (1998). Echoes of echoes? An episodic theory of lexical access. Psychological Review, 105, 251-279.

Goldinger, S. D., \& Azuma, T. (2004). Episodic memory reflected in printed word naming. Psychonomic Bulletin \& Review, 11, 716-722.

Grant, E. R., \& SpIvey, M. J. (2003). Eye movements and problem solving: Guiding attention guides thought. Psychological Science, 14, 462-466.

Hegarty, M. (2004). Mechanical reasoning by mental simulation. Trends in Cognitive Sciences, 8, 280-285.

Hintzman, D. L. (1986). "Schema abstraction" in a multiple-trace memory model. Psychological Review, 93, 411-428.

Kalish, M. L., Lewandowsky, S., \& Davies, M. (2005). Error-driven knowledge restructuring in categorization. Journal of Experimental Psychology: Learning, Memory, \& Cognition, 31, 846-861.

KaschaK, M. P., \& Glenberg, A. M. (2000). Constructing meaning: The role of affordances and grammatical constructions in sentence comprehension. Journal of Memory \& Language, 43, 508-529.

Logan, G. D. (2002). An instance theory of attention and memory. Psychological Review, 109, 376-400.

Newell, A. (1990). Unified theories of cognition. Cambridge, MA: Harvard University Press.

SIEGLER, R. S., \& ARAYA, R. (2005). A computational model of conscious and unconscious strategy discovery. In R. Kail (Ed.), Advances in child development and behavior (Vol. 33, pp. 1-42). Oxford: Elsevier.

Singer, J. D., \& Willett, J. B. (2003). Applied longitudinal data analysis. New York: Oxford University Press.

Solomon, K. O., \& Barsalou, L. W. (2001). Representing properties locally. Cognitive Psychology, 43, 129-169.

ZWAAN, R. A., \& TAYLOR, L. J. (2006). Seeing, acting, understanding: Motor resonance in language comprehension. Journal of Experimental Psychology: General, 135, 1-11.

\section{NOTES}

1. The present study provides additional evidence on this issue. If participants' force-tracing actions serve to trigger transfer from another domain, then those participants who possess a more abstract representation of alternation should transfer after relatively few force-tracing actions. These participants would be expected to generalize alternation easily, given that their representation was more abstract. Conversely, if participants are constructing a representation of alternation from their force-tracing actions, then the number of actions contributing to that representation should predict its generalizability.

2. The total sample size, including both left- and right-censored cases, was 173 . Left-censored cases were participants who used alternation on the first trial $(n=52)$; predictors cannot be computed for these cases. Right-censored cases were participants who did never used alternation during the experiment $(n=45)$. Thus, one reason for using existing data was that a very large sample is required to obtain sufficient "discovers" for modeling (postdiscovery) generalization.

3. Alternation, force-tracing, and the other strategies were coded from participants' videotaped verbalizations and motions; reliability among 5 coders was high ( $96 \%$ perfect agreement).

4. If a participant performed alternating actions on the current trial, the episodic traces of the participant's prior alternating actions and his or her episodic traces of prior "echos" (from previous recall experiences) contributed to the current echo. Thus, the number of action traces in episodic memory on participant i's discovery trial was computed as

$$
\text { Action traces }_{i, d \text { trial }}=\sum_{j=1}^{d \text { trial-1 }}\left[\mathrm{G}_{i j}+\sum_{k=1}^{j-1}\left(\text { action traces }_{i k}\right)\right] \text {. }
$$

$\mathrm{G}_{i j}$ is the number of gears correctly traced by participant $\mathrm{i}$ on trial $\mathrm{j}$, dtrial is the trial on which alternation was discovered, and $k$ indexes the actions traces that have accumulated across all previous trials. The effects reported here are most robust under the assumption that echos from 
episodic memory are also stored as episodic traces, however, the same pattern of results obtain without this assumption. All predictors were mean-centered to remove nonessential co-linearity, thereby facilitating interpretation of lower-order terms when interactions were included in the model.

5. $-2 \mathrm{LL}$ is the deviance statistic, distributed as chi-square. Significant change in the $-2 \mathrm{LL}$ indicates a reliable improvement in the fit of the model.

6. Adding accuracy (i.e., proportion correct across all previous trials) to the model did not contribute significantly to the fit, $-2 \operatorname{LL}\left[\chi^{2}(1)=\right.$ $2.07]$, nor did it appreciably change the parameter estimates above; all the model parameters take nearly identical values and remain significant. Likewise, including the timing of discovery (i.e., the trial number on which alternation was discovered), or the current trial number did not improve the model fit, $-2 \operatorname{LL}\left[\chi^{2} \mathrm{~s}(1)=.57, .03\right]$, or alter the estimated effects. Although generalizing more than once is possible, we focus on participants' first generalization to maximize power and avoid the complexities of analyzing repeated events.

(Manuscript received January 10, 2006; revision accepted for publication October 8, 2006.) 